\title{
Prediction of Early Recanalization after Intravenous Thrombolysis in Patients with Large-Vessel Occlusion
}

\author{
Young Dae Kim, ${ }^{a}$ Hyo Suk Nam, ${ }^{a}$ Joonsang Yoo, ${ }^{\text {bcc }}$ Hyungjong Park, ${ }^{\text {a,b }}$ Sung-Il Sohn, ${ }^{\mathrm{b}}$ Jeong-Ho Hong, \\ Byung Moon Kim, ${ }^{\mathrm{d}}$ Dong Joon Kim, ${ }^{\mathrm{d}}$ Oh Young Bang, ${ }^{\mathrm{e}}$ Woo-Keun Seo, ${ }^{\mathrm{e}}$ Jong-Won Chung, ${ }^{\mathrm{e}}$ Kyung-Yul Lee, ${ }^{\mathrm{f}}$ \\ Yo Han Jung, ${ }^{\mathrm{f}, \mathrm{g}}$ Hye Sun Lee, ${ }^{\mathrm{h}}$ Seong Hwan Ahn, ${ }^{\mathrm{i}}$ Dong Hoon Shin, ${ }^{\mathrm{j}}$ Hye-Yeon Choi, ${ }^{\mathrm{k}}$ Han-Jin Cho, ${ }^{\mathrm{l}}$ \\ Jang-Hyun Baek, ${ }^{\text {m,n }}$ Gyu Sik Kim, ${ }^{o}$ Kwon-Duk Seo, ${ }^{\text {,p }}$ Seo Hyun Kim, ${ }^{\mathrm{q}}$ Tae-Jin Song, ${ }^{\text {,s }}$ Jinkwon Kim, ${ }^{\mathrm{c}, \mathrm{t}}$ \\ Sang Won Han, ${ }^{\mathrm{u}}$ Joong Hyun Park, ${ }^{\mathrm{u}}$ Sung Ik Lee, ${ }^{\mathrm{p}}$ JoonNyung Heo, ${ }^{\mathrm{a}}$ Jin Kyo Choi, ${ }^{\mathrm{a}, \mathrm{d}}$ Ji Hoe Heo, ${ }^{\mathrm{a}}$ \\ on behalf of the Thrombus Imaging Study Group and the SECRET Study Group
}

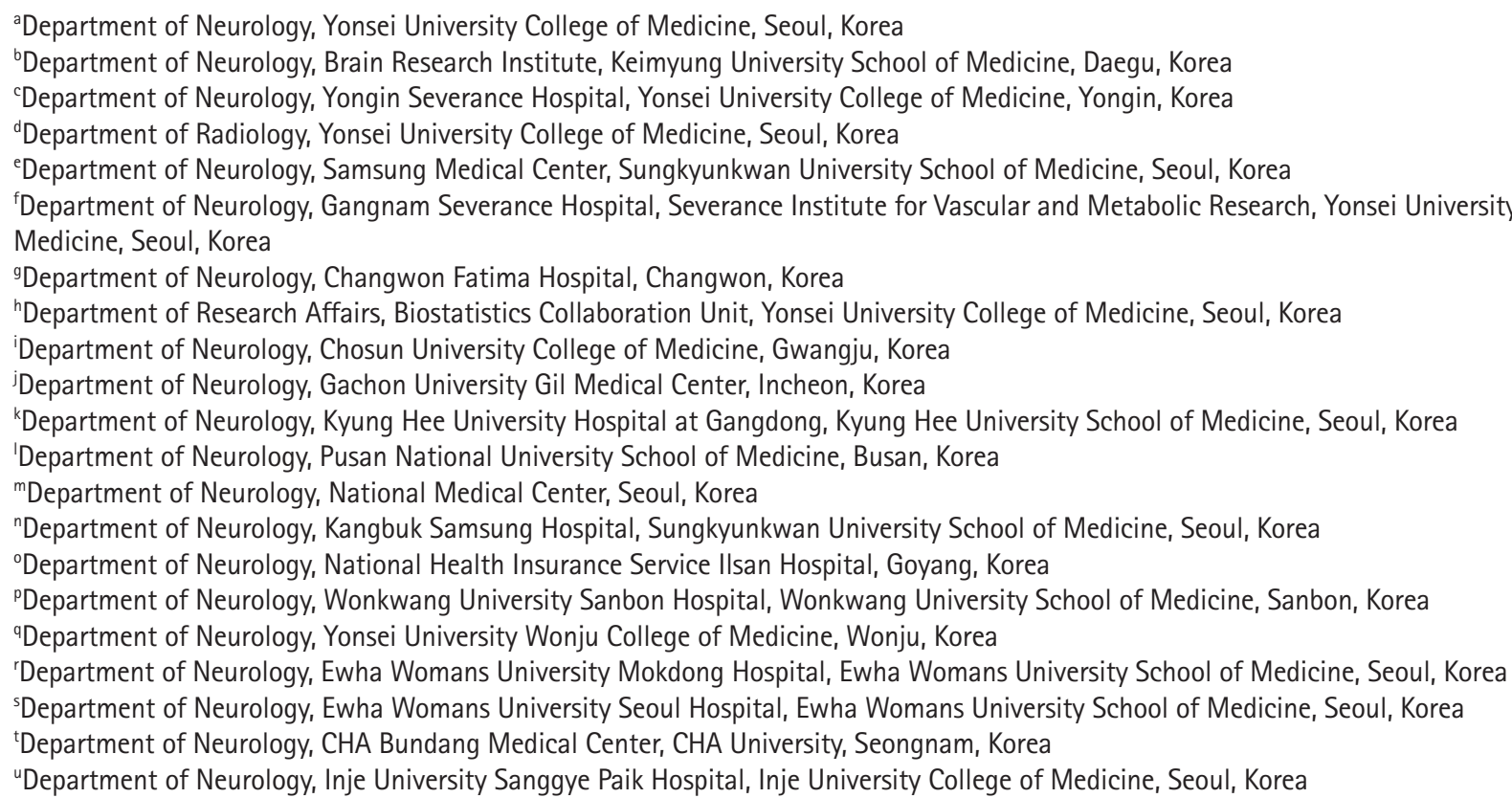

Background and Purpose We aimed to develop a model predicting early recanalization after intravenous tissue plasminogen activator (t-PA) treatment in large-vessel occlusion.

Methods Using data from two different multicenter prospective cohorts, we determined the factors associated with early recanalization immediately after t-PA in stroke patients with large-vessel occlusion, and developed and validated a prediction model for early recanalization. Clot volume was semiautomatically measured on thin-section computed tomography using software, and the degree of collaterals was determined using the Tan score. Follow-up angiographic studies were performed immediately after t-PA treatment to assess early recanalization.

Results Early recanalization, assessed $61.0 \pm 44.7$ minutes after t-PA bolus, was achieved in 15.5\% $(15 / 97)$ in the derivation cohort and in $10.5 \%$ (8/76) in the validation cohort. Clot volume (odds ratio [OR], $0.979 ; 95 \%$ confidence interval $[\mathrm{Cl}], 0.961$ to $0.997 ; P=0.020)$ and good collaterals $(\mathrm{OR}$,
Correspondence: Ji Hoe Heo Department of Neurology, Yonsei University College of Medicine, 50-1 Yonsei-ro, Seodaemun-gu, Seoul 03722, Korea

Tel: +82-2-2228-1605

Fax: +82-2-393-0705

E-mail:jhheo@yuhs.ac https://orcid.org/0000-0001-9898-3321

Received: August 27, 2020 Revised: January 17, 2021 Accepted: February 10, 2021 
$6.129 ; 95 \% \mathrm{Cl}, 1.592$ to $23.594 ; P=0.008)$ were significant factors associated with early recanalization. The area under the curve (AUC) of the model including clot volume was $0.819(95 \% \mathrm{Cl}$, 0.720 to 0.917$)$ and $0.842(95 \% \mathrm{Cl}, 0.746$ to 0.938$)$ in the derivation and validation cohorts, respectively. The AUC improved when good collaterals were added (derivation cohort: AUC, 0.876; $95 \% \mathrm{Cl}, 0.802$ to $0.950 ; P=0.164$; validation cohort: $\mathrm{AUC}, 0.949 ; 95 \% \mathrm{Cl}, 0.886$ to $1.000 ; P=0.036$ ). The integrated discrimination improvement also showed significantly improved prediction (0.097; $95 \% \mathrm{Cl}, 0.009$ to $0.185 ; P=0.032$ ).

Conclusions The model using clot volume and collaterals predicted early recanalization after intravenous t-PA and had a high performance. This model may aid in determining the recanalization treatment strategy in stroke patients with large-vessel occlusion.

Keywords Ischemia; Stroke; Thrombosis; Thrombolysis; Reperfusion

\section{Introduction}

Successful recanalization in acute stroke patients with large-vessel occlusion (LVO) is a main target of reperfusion therapy. To achieve early and complete successful recanalization, early treatment with either intravenous tissue plasminogen activator (IV t-PA) or endovascular treatment (EVT) is needed. ${ }^{1,2}$

Even after the successful introduction of EVT, bridging therapy with IV t-PA is recommended before EVT. ${ }^{2}$ However, IV t-PA treatment has low successful recanalization rates in patients with LVO. ${ }^{3-5}$ In addition, bridging therapy with IV t-PA before EVT may delay the initiation of EVT or increase the risk of bleeding complications, which may lead to unfavorable outcomes. ${ }^{6}$ In contrast, early recanalization that can be achieved with IV t-PA may be beneficial for outcomes. ${ }^{7}$ Although bridging therapy with IV t-PA is currently a debated issue, ${ }^{8}$ a specific group of patients may benefit from IV t-PA before EVT and selection of good responders would be helpful for improving patient outcomes.

A large clot burden determined either quantitatively or qualitatively is strongly associated with successful recanalization after IV t-PA treatment..$^{5,-13}$ In our previous study, almost all patients with thrombus measuring $>200 \mathrm{~mm}^{3}$ did not achieve early recanalization after IV t-PA; these patients were suggested to be the primary candidates for direct EVT. ${ }^{5}$ However, many patients had thrombus measuring $<200 \mathrm{~mm}^{3}$, and approximately $70 \%$ of them still did not achieve early recanalization. Furthermore, predicting patients who might achieve early recanalization after IV t-PA would be helpful for deciding IV t-PA treatment in those with LVO. This suggests the necessity of an improved model that predicts not only nonrecanalization but also successful recanalization.

While a clot burden is associated with early recanalization, the collateral status may also have a role. ${ }^{14}$ The presence of good collaterals is an indicator of a favorable outcome in patients undergoing recanalization treatment. ${ }^{15}$ Although aiding in keeping the penumbra area durable until achieving recanalization is the main reason, an enhanced thrombolytic effect by delivering more t-PA to the clot via backflow may also contribute to the favorable outcome. ${ }^{14,16}$ Therefore, we aimed to determine the predictors of early recanalization after IV t-PA treatment, including both clot volume and the degree of collaterals. We also aimed to develop and validate a simple prediction model for early recanalization using data from two different prospective cohorts.

\section{Methods}

\section{Study population}

We used data from two different multicenter stroke registries for developing a prediction model. For the derivation cohort, we used data from the Thrombus Imaging Study registry, which was a multicenter prospective study designed to provide clinical evidence for thrombus imaging for outcome prediction in reperfusion therapy. ${ }^{5}$ The inclusion criteria were treatment with IV t-PA within 4.5 hours of symptom onset and visible thrombi at the occlusion site of the distal internal carotid artery (ICA) and/or middle cerebral artery (MCA) on thin-section noncontrast computed tomography (NCCT). Patients were consecutively enrolled between February 2016 and August 2017 from nine hospitals. ${ }^{5}$ In this analysis, we included 97 patients with visible thrombus at the distal ICA or MCA (Figure 1).

For the validation cohort, we used data from the Selection Criteria in Endovascular Thrombectomy and Thrombolytic Therapy (SECRET) registry (Clinicaltrials.gov: NCT02964052). ${ }^{17}$ The SECRET registry is a nationwide multicenter registry for exploring the selection criteria for patients who would benefit from reperfusion therapies. This study included 1,026 patients who 

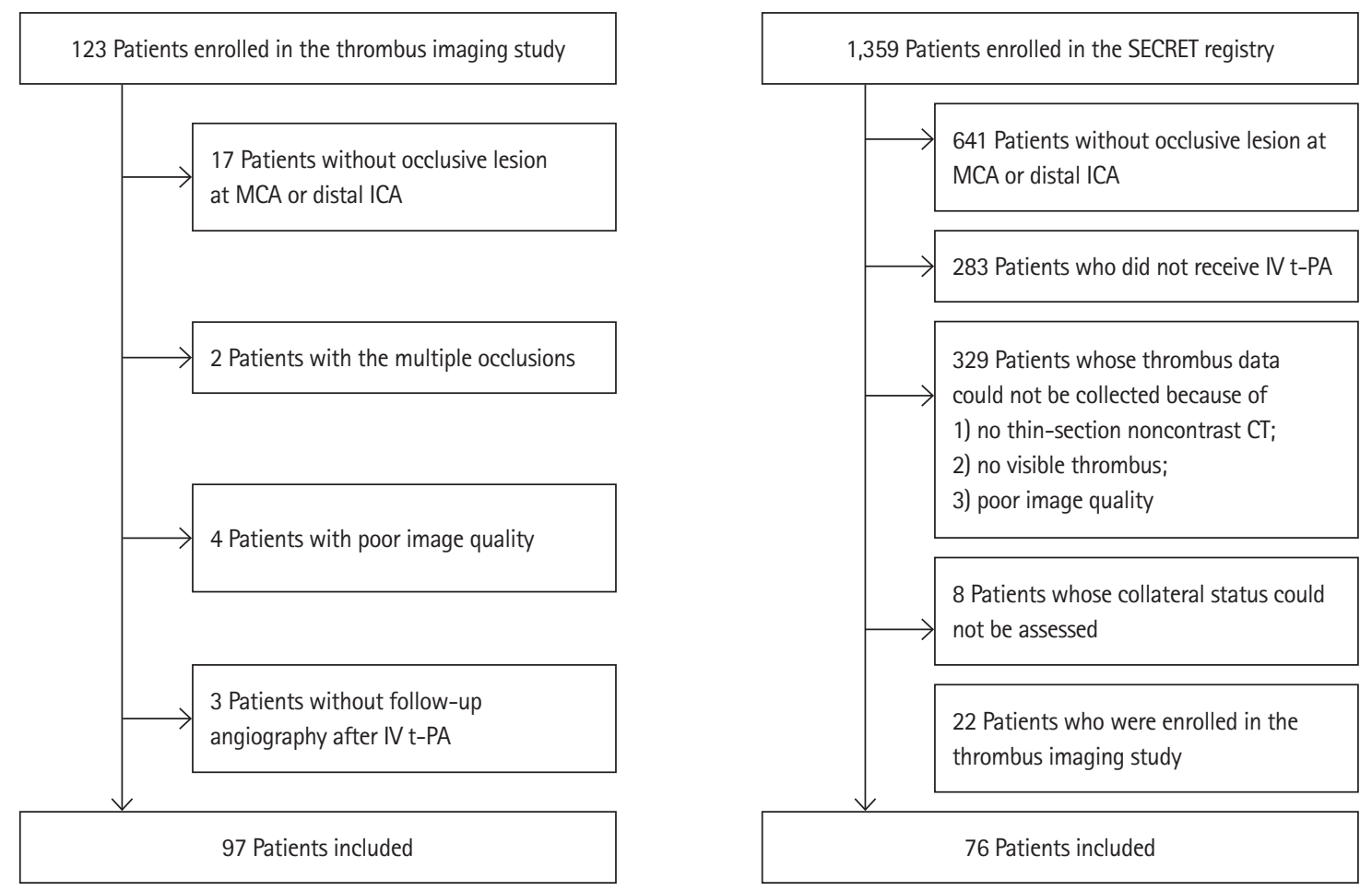

A

Figure 1. Patient selection: (A) derivation cohort, (B) validation cohort. MCA, middle cerebral artery; ICA, internal carotid artery; IV t-PA, intravenous tissue plasminogen activator; SECRET, Selection Criteria in Endovascular Thrombectomy and Thrombolytic Therapy; CT, computed tomography.

had been registered retrospectively from 15 hospitals between January 2012 and December 2015 and 333 patients who had been registered prospectively from 13 hospitals between November 2016 and December 2017. There were 98 patients with visible thrombi in the distal ICA and/or MCA on initial thin-section NCCT and those with imaging data available before and after IV t-PA. However, there was an overlap of the study period between the Thrombus Imaging Study and the SECRET study. Therefore, we excluded 22 patients (anonymized) who were enrolled during the overlapped period. Finally, 76 patients were included for the validation (Figure 1). This study was approved by the Institutional Review Board of each study hospital, and written informed consent was obtained from all patients enrolled prospectively.

\section{Imaging protocol and reperfusion therapy}

The patients included in this study underwent thin-section (1 or $1.25 \mathrm{~mm}$ ) NCCT and computed tomography angiography (CTA) before IV t-PA. All patients were treated with IV t-PA (Actilyse, Boehringer-Ingelheim, Ingelheim, Germany) at a standard dose of $0.9 \mathrm{mg} / \mathrm{kg}$ (10\% as a bolus followed by $90 \%$ as an infusion for 60 minutes) within 4.5 hours of stroke onset. The inclusion and exclusion criteria for IV t-PA treatment were based on the guidelines, which were not different among the study hospitals. Further EVT was sequentially performed if the patients did not show clinical improvement or did not achieve arterial recanalization after IV t-PA treatment. Follow-up CTA or magnetic resonance angiography (MRA) was immediately performed after the end of IV t-PA if the patients were not treated with EVT.

\section{Assessment of imaging findings}

The volume and density of thrombus were semiautomatically measured on baseline thin-section NCCT using three-dimensional software (Xelis, Infinitt, Seoul, Korea). The detailed methods have been previously described. ${ }^{5,18,19}$ Briefly, when a hyperdense artery (pixels between 50 and 100 Hounsfield units) was identified on thin-section NCCT, this thrombus area was automatically shown as a red area. When clicking any portion within the thrombus area and subsequently the "Dilate" icon, every slice of thrombus was merged with automatic pixel dilation and region growing. Thereafter, the volume and density of the thrombus were shown on a screen within 1 minute by automatic calculation. Simultaneously, CTA maximum-intensity projection images and thin-section NCCT images could be synchronized on a screen, which could enable the easy identification of thrombus.

The presence of early recanalization was assessed on CTA or 
MRA images taken immediately at the end of IV t-PA or on digital subtraction angiography images taken during EVT. Successful recanalization was defined as a modified Thrombolysis in Cerebral Infarction (TICl) grade of $2 b$ or 3 on digital subtraction angiography ${ }^{20}$ or an arterial occlusive lesion score of 3 on CTA or MRA. We also determined the degree of collaterals using the Tan collateral score ${ }^{11,16}$ based on the single-phase CTA axial image performed before IV t-PA treatment, and the presence of good collaterals was defined as a Tan score of 3 . We also collected the Alberta Stroke Program Early CT score. Two stroke neurologists (Y.D.K. and J.Y.) measured the thrombus volume and density and determined the Tan score, Alberta Stroke Program Early CT score, and $\mathrm{TICl}$ grade. Entire imaging analyses were independently performed without knowledge of any clinical information. In the case of discrepancies, a consensus was reached for the derivation cohort and adjudicated by the imaging adjudication committee for the validation cohort.

\section{Clinical variables}

We collected data on demographics, risk factors, and premorbid disability (dependent status defined as a modified Rankin Scale score of 3-5). Time parameters such as the intervals from stroke onset to initial brain imaging, from onset to IV t-PA, and from the initiation of IV t-PA to follow-up angiographic studies were determined. Data on the total dose of t-PA for each patient were collected. The stroke severity was assessed using the National Institutes of Health Stroke Scale.

\section{Statistical analyses}

Data are presented as frequency (percentage) for categorical variables and mean \pm standard deviation or median (interquartile range) for continuous variables. Differences between the groups were assessed using Student's t-test or the Mann-Whitney U-test for continuous variables and the chi-square test or Fisher's exact test for categorical variables, as appropriate.

Univariable and multivariable logistic regression analyses were performed to determine the independent factors for early successful recanalization after IV t-PA. The collinearity of combinations of variables in the derivation cohort was evaluated using variation inflation factors $(<2$ being considered nonsignificant). Odds ratios (ORs) with 95\% confidence intervals (Cls) for each variable included in the model were finally calculated. On the basis of the results of multivariable logistic regression analysis, we developed a prediction model and a nomogram.

We compared the predictive ability between the model including clot volume only and the model in which the presence of good collaterals was added to clot volume by computing the area under the curve (AUC) using the Delong method. The integrated discrimination improvement (IDI) index was computed to investigate whether adding the presence of good collaterals to clot volume would improve the predictive power. We also plotted decision curves to assess the net benefit of nomogram-assisted decisions.

We examined the calibration (calibration plots and Hosmer-Lemeshow test) and discrimination (AUC). To assess the external validity of model performance, we applied our prediction model to the validation cohort. We examined the performance of the final model both in the derivation and external validation cohorts in terms of discrimination using AUC and in terms of calibration by plotting the agreement between the predicted and observed probabilities across the quartiles of scores. In addition, we determined the optimal cutoff value of the prediction value based on nomogram, which was verified by calculating the Youden index.

Statistical analyses were performed using R software package version 3.6.2 (http://www.R-project.org). All statistical tests were two-tailed; $P<0.05$ was considered statistically significant, whereas $0.05 \leq P<0.2$ was considered a trend toward significance to increase the sensitivity for detecting potential selection bias.

\section{Results}

\section{Baseline characteristics}

Follow-up angiographic studies were performed at a mean of $61.0 \pm 44.7$ minutes after the bolus injection of t-PA $(65.1 \pm 49.1$ minutes in the derivation cohort and $55.9 \pm 38.1$ minutes in the validation cohort, $P=0.181$ ). The baseline characteristics including clot volume and the frequency of good collaterals were not different between the derivation and validation cohorts, except diabetes and dyslipidemia, which had lower frequencies in the derivation cohort (Table 1).

\section{Determinants for successful recanalization}

The occluded artery was recanalized immediately after IV t-PA in 15.5\% (15/97) of the derivation cohort and in 10.5\% (8/76) of the validation cohort. The factors associated with early recanalization in the derivation cohort were smaller clot volumes, good collaterals, and lower National Institutes of Health Stroke Scale scores on univariable analysis. Multivariable logistic regression analysis showed that clot volume (OR, 0.979 per 1 $\mathrm{mm}^{3} ; 95 \% \mathrm{Cl}, 0.961$ to $\left.0.997 ; P=0.02\right)$ and good collaterals $(\mathrm{OR}, 6.129 ; 95 \% \mathrm{Cl}, 1.592$ to $23.594 ; P=0.008)$ were independent and significant predictors of early recanalization (Table 2). These findings were still observed when we divided the study 
Table 1. Baseline characteristics of the derivation and external validation cohorts

\begin{tabular}{|c|c|c|c|}
\hline Characteristic & Derivation cohort $(n=97)$ & Validation cohort $(n=76)$ & $P$ \\
\hline Age $(\mathrm{yr})$ & $67.9 \pm 12.8$ & $66.9 \pm 10.8$ & 0.620 \\
\hline Male sex & $44(45.4)$ & $39(51.3)$ & 0.437 \\
\hline Hypertension & $58(59.8)$ & $54(71.1)$ & 0.124 \\
\hline Diabetes & 27 (27.8) & $40(52.6)$ & 0.001 \\
\hline Dyslipidemia & $28(28.9)$ & $34(44.7)$ & 0.031 \\
\hline Atrial fibrillation & $46(47.4)$ & $46(60.5)$ & 0.086 \\
\hline Previous stroke & $15(15.5)$ & 15 (19.7) & 0.461 \\
\hline Premorbid disability & $1(1.0)$ & $3(3.9)$ & 0.321 \\
\hline Sequential endovascular treatment & $73(75.3)$ & $59(77.6)$ & 0.716 \\
\hline Occlusion site & & & 0.727 \\
\hline Distal ICA $\pm \mathrm{M} 1$ & $21(21.6)$ & $20(26.3)$ & \\
\hline MCA M1 & $59(60.8)$ & $42(55.3)$ & \\
\hline MCA M2 & 17 (17.5) & $14(18.4)$ & \\
\hline Initial NIHSS score & $15(11-18)$ & $14(9-19)$ & 0.736 \\
\hline ASPECT score & $8(7-9)$ & $8(7-9)$ & 0.306 \\
\hline $\mathrm{t}-\mathrm{PA}$ dose $(\mathrm{mg})$ & $54.3 \pm 12.7$ & $54 \pm 10$ & 0.887 \\
\hline Onset to t-PA & $123.1 \pm 66.2$ & $123.4 \pm 80.1$ & 0.974 \\
\hline Onset to CT & $102.3 \pm 65.7$ & $100.2 \pm 78.4$ & 0.843 \\
\hline t-PA to follow-up imaging & $65.1 \pm 49.1$ & $55.9 \pm 38.1$ & 0.181 \\
\hline Thrombus volume $\left(\mathrm{mm}^{3}\right)$ & $124.7 \pm 102.1$ & $144.3 \pm 122.8$ & 0.253 \\
\hline Thrombus HU & $51.5 \pm 3.5$ & $52.6 \pm 3.6$ & 0.038 \\
\hline Good collaterals, 3 vs. $0-2$ & 31 (32.0) & 15 (19.7) & 0.071 \\
\hline
\end{tabular}

Values are presented as mean \pm standard deviation, number (\%), or median (interquartile range).

ICA, internal carotid artery; MCA, middle cerebral artery; NIHSS, National Institutes of Health Stroke Scale; ASPECT, Alberta Stroke Program Early Computed Tomography; t-PA, tissue plasminogen activator; $\mathrm{CT}$, computed tomography; $\mathrm{HU}$, Hounsfield unit.

Table 2. Univariable and multivariable analyses for early recanalization

\begin{tabular}{|c|c|c|c|c|}
\hline \multirow{2}{*}{ Variable } & \multicolumn{2}{|c|}{ Univariable analysis } & \multicolumn{2}{|c|}{ Multivariable analysis } \\
\hline & Odds ratio $(95 \% \mathrm{Cl})$ & $P$ & Odds ratio (95\% Cl) & $P$ \\
\hline Age & 0.968 (0.929-1.008) & 0.117 & & \\
\hline Male sex & $0.496(0.162-1.524)$ & 0.221 & & \\
\hline Hypertension & $3.130(0.821-11.934)$ & 0.095 & & \\
\hline Diabetes & $1.364(0.419-4.435)$ & 0.606 & & \\
\hline Dyslipidemia & $0.570(0.148-2.198)$ & 0.414 & & \\
\hline Atrial fibrillation & $0.700(0.228-2.146)$ & 0.533 & & \\
\hline Previous stroke & 2.347 (0.634-8.688) & 0.201 & & \\
\hline Premorbid disability & $0.000(0.000-N A)$ & 1.000 & & \\
\hline Initial NIHSS score & $0.882(0.797-0.976)$ & 0.015 & 0.963 (0.852-1.089) & 0.551 \\
\hline ASPECT score & $1.458(0.933-2.279)$ & 0.098 & & \\
\hline t-PA dose $(\mathrm{mg})$ & $1.010(0.965-1.056)$ & 0.670 & & \\
\hline Onset to t-PA & 0.999 (0.991-1.008) & 0.886 & & \\
\hline Onset to CT & 1.000 (0.992-1.009) & 0.928 & & \\
\hline t-PA to follow-up imaging & $1.004(0.995-1.013)$ & 0.394 & & \\
\hline Clot volume $\left(\mathrm{mm}^{3}\right)$ & $0.976(0.961-0.992)$ & 0.004 & $0.979(0.961-0.997)$ & 0.020 \\
\hline Clot HU & $0.873(0.744-1.023)$ & 0.094 & & \\
\hline Good collateral, 3 vs. $0-2$ & $8.525(2.441-29.769)$ & 0.001 & $6.129(1.592-23.594)$ & 0.008 \\
\hline
\end{tabular}

$\mathrm{Cl}$, confidence interval; NA, not applicable; NIHSS, National Institutes of Health Stroke Scale; ASPECT, Alberta Stroke Program Early Computed Tomography; t-PA, tissue plasminogen activator; $\mathrm{CT}$, computed tomography; HU, Hounsfield unit. 
population into two groups based on the timing ( $\geq 45$ or $\leq 75$ minutes) of follow-up angiographic studies after IV t-PA (Supplementary Table 1).

As the clot volume was a strong factor associated with early recanalization, we determined whether adding good collaterals to the prediction model would improve the model performance. When the presence of good collaterals was added to clot volume, the AUC improved from 0.819 ( $95 \% \mathrm{Cl}, 0.720$ to 0.917$)$ to $0.876(95 \% \mathrm{Cl}, 0.802$ to 0.950$)$ in the derivation cohort $(P=0.164)$ (Figure 2A) and from $0.842(95 \% \mathrm{Cl}, 0.746$ to 0.938$)$ to 0.949 (95\% Cl, 0.886 to 1.000$)$ in the validation cohort $(P=0.036)$. The decision curves for the probability of successful recanalization in the derivation cohort showed better performance when the presence of good collaterals was added to clot volume (Figure 2B). The computation of IDI also showed significantly improved prediction (IDI, 0.097; 95\% Cl, 0.009 to $0.185 ; P=0.032$ ). The Hosmer-Lemeshow tests were not significant in the derivation cohort $(P=0.970)$ and the validation cohort $(P=0.935)$, which means a good fit. Figure 3 demonstrates the predicted versus observed probability based on the quartile of the scores, which showed good correlation.

Accordingly, we developed a model predicting early recanalization after IV t-PA treatment using both clot volume and good collaterals. For easy use of the prediction model, we also developed a nomogram predicting early recanalization by assigning a graphic score to each of clot volume and good collaterals, with a point range from 0 to 20 . The scores were summed to generate a total score, which was finally converted into an individual probability of early successful recanalization after IV
t-PA (Figure 4). Higher total points based on the sum of the assigned number of points for good collaterals and clot volume were associated with the chance of early recanalization (Supplementary Figure 1). The cut off value of $>0.1107$ of the prediction model value for predicting early recanalization, which was based on the Youden index, had good sensitivity (100\%) and specificity (68.3\%).

\section{Discussion}

In the present study, early recanalization after IV t-PA was significantly associated with clot volume and collateral status (thus, the model was named " $2 \mathrm{C}^{\prime}$ ). The prediction model using both clot volume and good collaterals could reliably predict early recanalization after IV t-PA treatment among patients with LVO. This $2 \mathrm{C}$ model was externally validated using another population enrolled in a nationwide multicenter registry. We also developed a nomogram using these two variables for rapid and easy use in clinical practice, which can help identify patients who would be expected to achieve early recanalization after IV t-PA treatment.

With EVT becoming the standard treatment, bridging therapy with IV t-PA before EVT in patients with LVO is a debated issue. ${ }^{8}$ This is because, in patients with LVO, recanalization is achieved immediately after t-PA in only about $10 \%$ to $15 \% \%^{4,5,13,14,19,21}$ and t-PA may increase the risk of bleeding and distal embolization of partially lysed clots. It may also delay the begin.

Recently, the Direct Intraarterial Thrombectomy in Order to
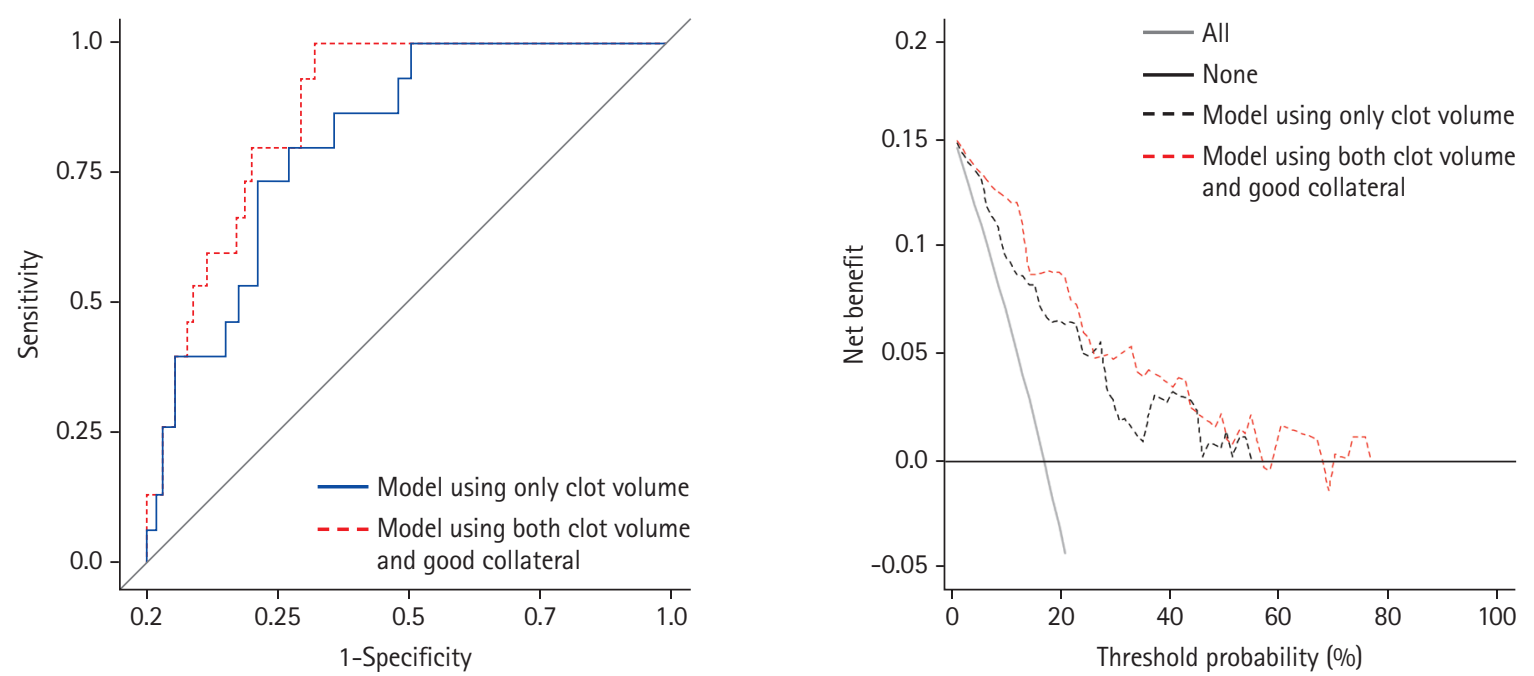

A

B

Figure 2. Comparison of receiver operating characteristic (ROC) curves between two models (A) and decision curve analysis (B). (A) ROC curves of the derivation cohorts and area under the curve (AUC) values (model using only clot volume, 0.819; model using both clot volume and good collaterals, 0.876). (B) Decision curve analysis showing that the model using both clot volume and good collaterals was the preferred model. 

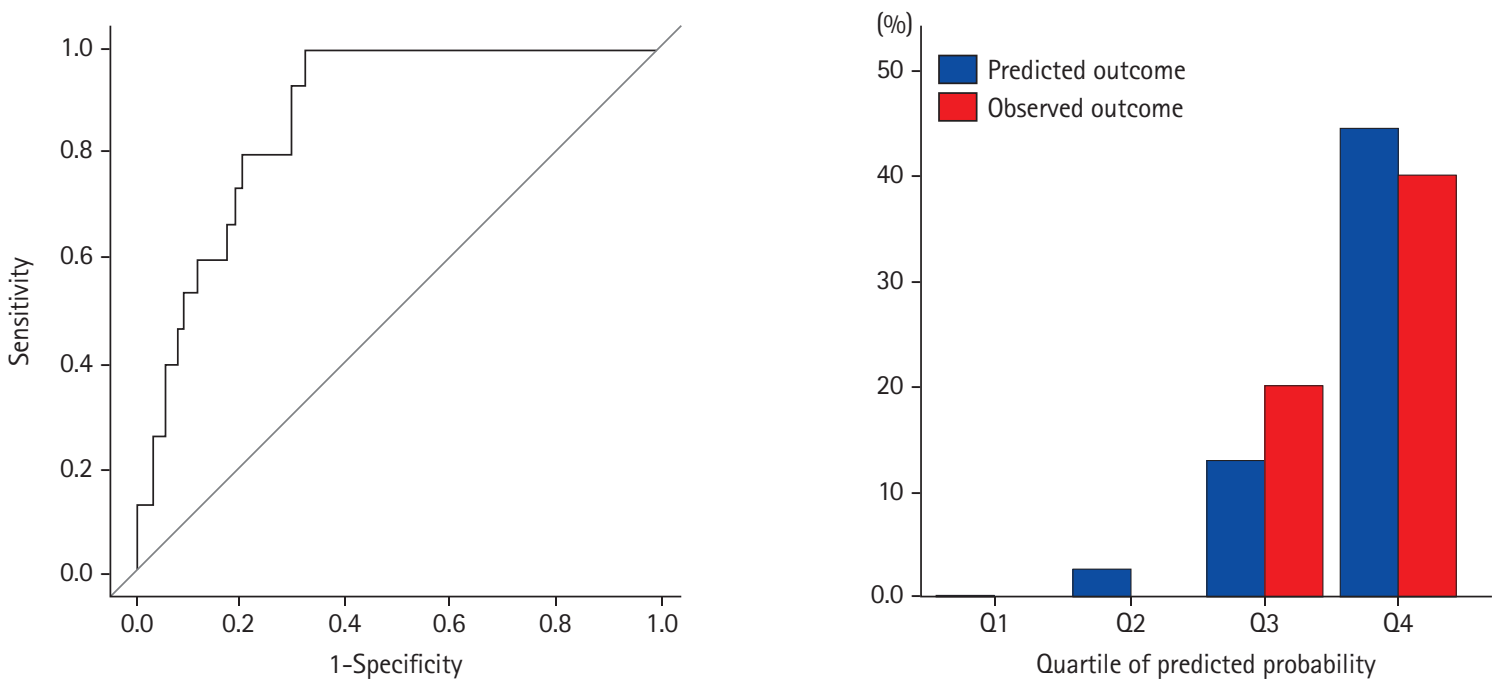

A

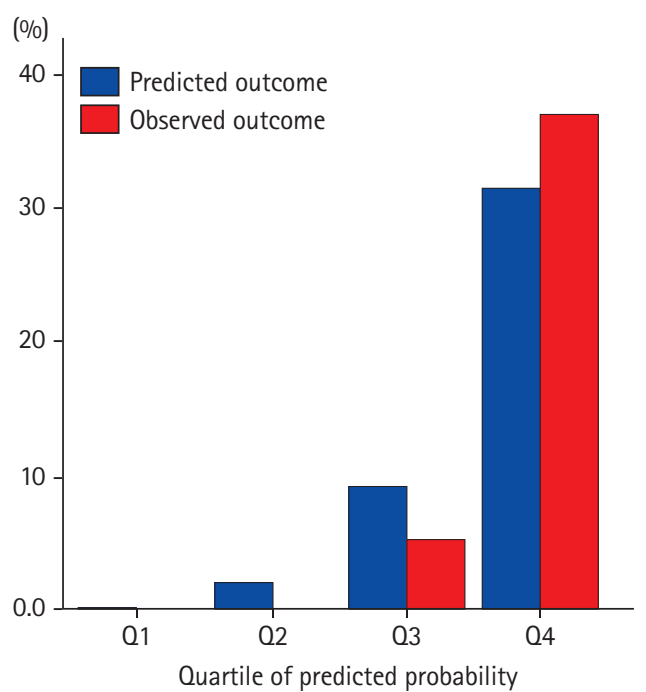

C

Figure 3. Assessment of discrimination and calibration in the derivation cohort ( $A, B)$ and the validation cohort (C, D). (A) Receiver operating characteristic (ROC) curves of the derivation cohort and area under the curve (AUC) values (AUC, 0.876; 95\% confidence interval [CI], 0.802 to 0.950). (B) Calibration plot per quartile of the scores in the derivation cohort. (C) ROC curves of the validation cohort and AUC values (AUC, 0.949; $95 \%$ Cl, 0.886 to 1.000 ). (D) Calibration plot per quartile of the scores in the validation cohort.

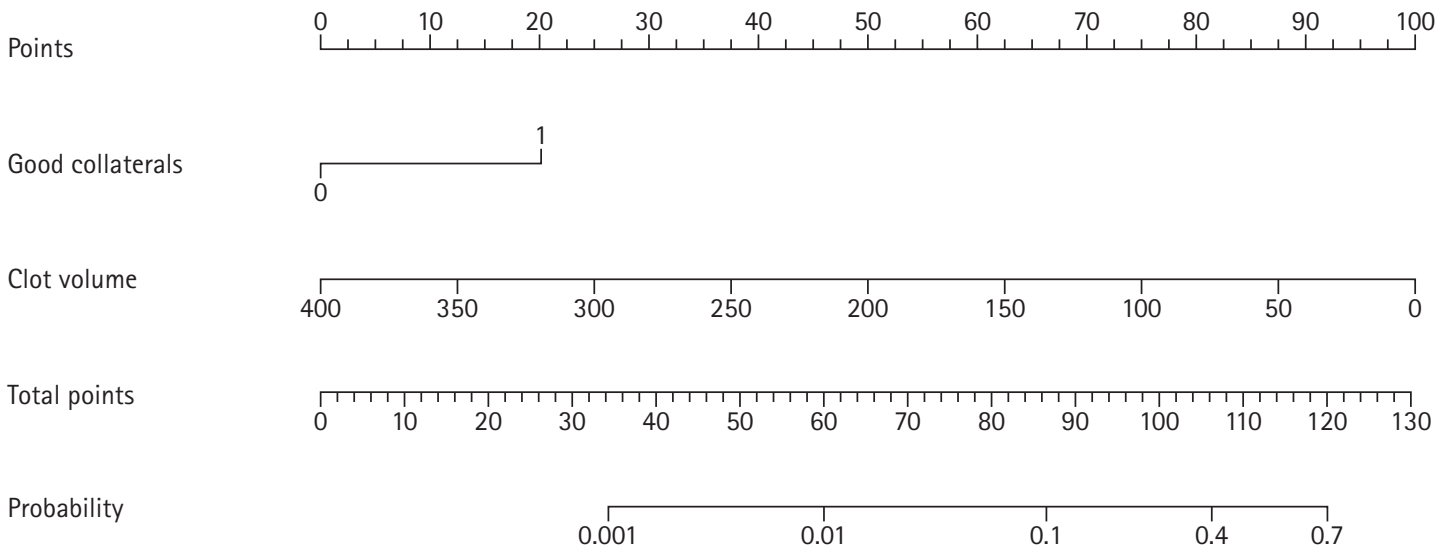

Figure 4. Nomogram for predicting successful recanalization within 1 hour after intravenous tissue plasminogen activator. 
Revascularize Acute Ischemic Stroke Patients with Large Vessel Occlusion Efficiently in Chinese Tertiary Hospitals: a Multicenter Randomized Clinical Trial (DIRECT-MT) demonstrated that EVT alone was noninferior to combined IV t-PA and EVT with respect to functional outcomes, but it was associated with lower rates of successful recanalization than the combined therapy. ${ }^{22}$ Although similar trials (NCT03192332, NCT03494920, and ISRCTN80619088) are ongoing, ${ }_{1}^{23,24}$ it is questionable whether one strategy (either direct EVT or combined intravenous thrombolysis and EVT) should be the only treatment option for all patients with LVO. A certain group of patients would benefit from IV t-PA before EVT, whereas direct EVT would be better in another group of patients. ${ }^{8}$ In this context, prediction of early recanalization after t-PA would be helpful for deciding the strategy between direct EVT and combined intravenous thrombolysis and EVT. For example, IV t-PA before EVT may be considered when the probability of early recanalization by IV t-PA is high based on the prediction model. When the probability is very low, direct EVT may be considered as IV t-PA may increase the risk of bleeding and lead to delay the initiation of EVT.

In t-PA treatment, clot volume is an important factor associated with early recanalization. This study showed that good collaterals also enhance the chance for early successful recanalization. In a previous study based on perfusion magnetic resonance imaging, good collaterals were associated with recanalization within 3 hours after t-PA treatment. ${ }^{14}$ Good collaterals can lead to a bidirectional action of t-PA (forward and back flow). This may be able to enhance the proteolytic action of t-PA by increasing the surface area contacting the t-PA. ${ }^{14,16,25}$

Although there have been several prognostic models or scoring systems for estimating safety or efficacy of thrombolytic therapy with IV t-PA for acute ischemic stroke, little is known on the model for predicting the successful recanalization by IV t-PA within 1 hour. In this study, recanalization was assessed about 60 minutes after IV t-PA injection, immediately after the end of IV t-PA infusion when making a decision regarding further EVT is necessary. Therefore, prediction of recanalization at this time point is important for determining the treatment strategy. We found that only two variables (clot volume and good collaterals) were sufficient for reliably predicting recanalization with high performance at this time point. The nomogram that we developed may also be helpful for making a rapid decision.

This study had some limitations. Although collaterals are easily determined on CTA, measurement of clot volume requires a software program. Therefore, the wide use of this prediction model is limited because it depends on the availability of the software. We only included patients with occlusion of the MCA or distal ICA. Thereby, the predictive ability of our model for occlusion at other cerebral arterial segments is unknown. In addition, although we tried to show the $2 \mathrm{C}$ model in this study could be useful for predicting the early recanalization, the rate of early recanalization was low. This might lead the relatively low statistical power. So, we thought further analysis including larger amount of data would be necessary.

\section{Conclusions}

We showed that clot volume and good collaterals were associated with early recanalization after IV t-PA treatment, and the prediction model using clot volume and good collaterals showed high performance in the derivation and validation cohorts. Our model may aid in the decision making with respect to the recanalization strategy.

\section{Supplementary materials}

Supplementary materials related to this article can be found online at https://doi.org/10.5853/jos.2020.03622.

\section{Disclosure}

The authors have no financial conflicts of interest.

\section{Acknowledgments}

This work was supported by a grant from the Basic Science Research Program through the National Research Foundation of Korea (NRF) funded by the Ministry of Education (NRF2018R1A2A3074996); a faculty research grant of Yonsei University College of Medicine (6-2020-0202); and the "Dongwha" Faculty Research Assistance Program of Yonsei University College of Medicine (6-2019-0191).

\section{References}

1. Goyal M, Menon BK, van Zwam WH, Dippel DW, Mitchell PJ, Demchuk AM, et al. Endovascular thrombectomy after large-vessel ischaemic stroke: a meta-analysis of individual patient data from five randomised trials. Lancet 2016;387: 1723-1731.

2. Powers WJ, Rabinstein AA, Ackerson T, Adeoye OM, Bambakidis NC, Becker K, et al. Guidelines for the early management of patients with acute ischemic stroke: 2019 update to the 2018 guidelines for the early management of acute isch- 
emic stroke. A guideline for healthcare professionals from the American Heart Association/American Stroke Association. Stroke 2019;50:e344-e418.

3. Lee KY, Han SW, Kim SH, Nam HS, Ahn SW, Kim DJ, et al. Early recanalization after intravenous administration of recombinant tissue plasminogen activator as assessed by preand post-thrombolytic angiography in acute ischemic stroke patients. Stroke 2007;38:192-193.

4. Campbell BCV, Mitchell PJ, Churilov L, Yassi N, Kleinig TJ, Dowling RJ, et al. Tenecteplase versus alteplase before thrombectomy for ischemic stroke. N Engl J Med 2018;378:1 573-1582.

5. Yoo J, Baek JH, Park H, Song D, Kim K, Hwang IG, et al. Thrombus volume as a predictor of nonrecanalization after intravenous thrombolysis in acute stroke. Stroke 2018;49:2108-2115.

6. Fischer U, Kaesmacher J, Mendes Pereira V, Chapot R, Siddiqui $A H$, Froehler MT, et al. Direct mechanical thrombectomy versus combined intravenous and mechanical thrombectomy in large-artery anterior circulation stroke: a topical review. Stroke 2017;48:2912-2918.

7. Tsivgoulis G, Saqqur M, Sharma VK, Brunser A, Eggers J, Mi-

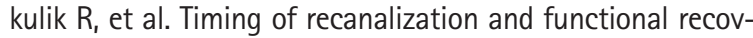
ery in acute ischemic stroke. J Stroke 2020;22:130-140.

8. Albers GW. Thrombolysis before thrombectomy: to be or DIRECT-MT? N Eng/ J Med 2020;382:2045-2046.

9. Zhu G, Michel P, Jovin T, Patrie JT, Xin W, Eskandari A, et al. Prediction of recanalization in acute stroke patients receiving intravenous and endovascular revascularization therapy. Int J Stroke 2015;10:28-36.

10. Rohan V, Baxa J, Tupy R, Cerna L, Sevcik P, Friesl M, et al. Length of occlusion predicts recanalization and outcome after intravenous thrombolysis in middle cerebral artery stroke. Stroke 2014:45:2010-2017.

11. Tan IY, Demchuk AM, Hopyan J, Zhang L, Gladstone D, Wong $\mathrm{K}$, et al. CT angiography clot burden score and collateral score: correlation with clinical and radiologic outcomes in acute middle cerebral artery infarct. AJNR Am J Neuroradiol 2009;30:525-531.

12. Heo JH, Kim K, Yoo J, Kim YD, Nam HS, Kim EY. Computed tomography-based thrombus imaging for the prediction of recanalization after reperfusion therapy in stroke. J Stroke 2017;19:40-49.

13. Behrens $L$, Möhlenbruch $M$, Stampfl $S$, Ringleb PA, Hametner $C$, Kellert $L$, et al. Effect of thrombus size on recanalization by bridging intravenous thrombolysis. Eur J Neurol 2014;21: 1406-1410.

14. Seners $P$, Roca $P$, Legrand $L$, Turc $G$, Cottier JP, Cho TH, et al.
Better collaterals are independently associated with post-thrombolysis recanalization before thrombectomy. Stroke 2019;50:867-872.

15. Derraz I, Bourcier R, Soudant M, Soize $S$, Hassen WB, Hossu $G$, et al. Does clot burden score on baseline T2*-MRI impact clinical outcome in acute ischemic stroke treated with mechanical thrombectomy? J Stroke 2019;21:91-100.

16. Bang OY, Goyal M, Liebeskind DS. Collateral circulation in ischemic stroke: assessment tools and therapeutic strategies. Stroke 2015;46:3302-3309.

17. Kim YD, Heo JH, Yoo J, Park H, Kim BM, Bang OY, et al. Improving the clinical outcome in stroke patients receiving thrombolytic or endovascular treatment in Korea: from the SECRET Study. J Clin Med 2020;9:717.

18. Kim EY, Lee SK, Kim DJ, Suh SH, Kim J, Heo JH, et al. Detection of thrombus in acute ischemic stroke: value of thin-section noncontrast-computed tomography. Stroke 2005;36: 2745-2747.

19. Kim YD, Nam HS, Kim SH, Kim EY, Song D, Kwon I, et al. Time-dependent thrombus resolution after tissue-type plasminogen activator in patients with stroke and mice. Stroke 2015;46:1877-1882.

20. Higashida RT, Furlan AJ, Roberts $H$, Tomsick T, Connors B, Barr J, et al. Trial design and reporting standards for intra-arterial cerebral thrombolysis for acute ischemic stroke. Stroke 2003;34:e109-e137.

21. Seners P, Caroff J, Chausson N, Turc G, Denier $C$, Piotin M, et al. Recanalization before thrombectomy in tenecteplase vs. alteplase-treated drip-and-ship patients. J Stroke 2019;21: 105-107.

22. Yang $P$, Zhang $Y$, Zhang $L$, Zhang $Y$, Treurniet $K M$, Chen $W$, et al. Endovascular thrombectomy with or without intravenous alteplase in acute stroke. N Engl J Med 2020;382:1981-1993.

23. Katsanos $A H$, Malhotra $K$, Goyal $N$, Arthur $A$, Schellinger PD, Köhrmann $\mathrm{M}$, et al. Intravenous thrombolysis prior to mechanical thrombectomy in large vessel occlusions. Ann Neurol 2019;86:395-406

24. Suzuki K, Kimura K, Takeuchi M, Morimoto M, Kanazawa R, Kamiya $Y$, et al. The randomized study of endovascular therapy with versus without intravenous tissue plasminogen activator in acute stroke with ICA and M1 occlusion (SKIP study). Int J Stroke 2019;14:752-755.

25. Zhang S, Zhang $X$, Yan S, Lai Y, Han Q, Sun J, et al. The velocity of collateral filling predicts recanalization in acute ischemic stroke after intravenous thrombolysis. Sci Rep 2016;6: 27880. 
Supplementary Table 1. Multivariable analyses for early recanalization and the area under curves of models by the timing of follow-up angiographic studies

\begin{tabular}{|c|c|c|c|c|}
\hline \multirow{2}{*}{ Variable } & \multicolumn{2}{|c|}{$\geq 45$ minutes } & \multicolumn{2}{|c|}{$\leq 75$ minutes } \\
\hline & OR (95\% Cl) & $P$ & OR $(95 \% \mathrm{Cl})$ & $P$ \\
\hline Clot volume $\left(\mathrm{mm}^{3}\right)$ & 0.980 (0.963-0.997) & 0.024 & $0.972(0.946-0.999)$ & 0.040 \\
\hline Good collaterals & $3.972(0.840-18.796)$ & 0.082 & 10.714 (1.790-64.139) & 0.009 \\
\hline AUC of model & \multicolumn{2}{|c|}{0.862} & \multicolumn{2}{|c|}{0.908} \\
\hline
\end{tabular}

$\mathrm{OR}$, odds ratio; $\mathrm{Cl}$, confidence interval; $\mathrm{AUC}$, area under the curve.

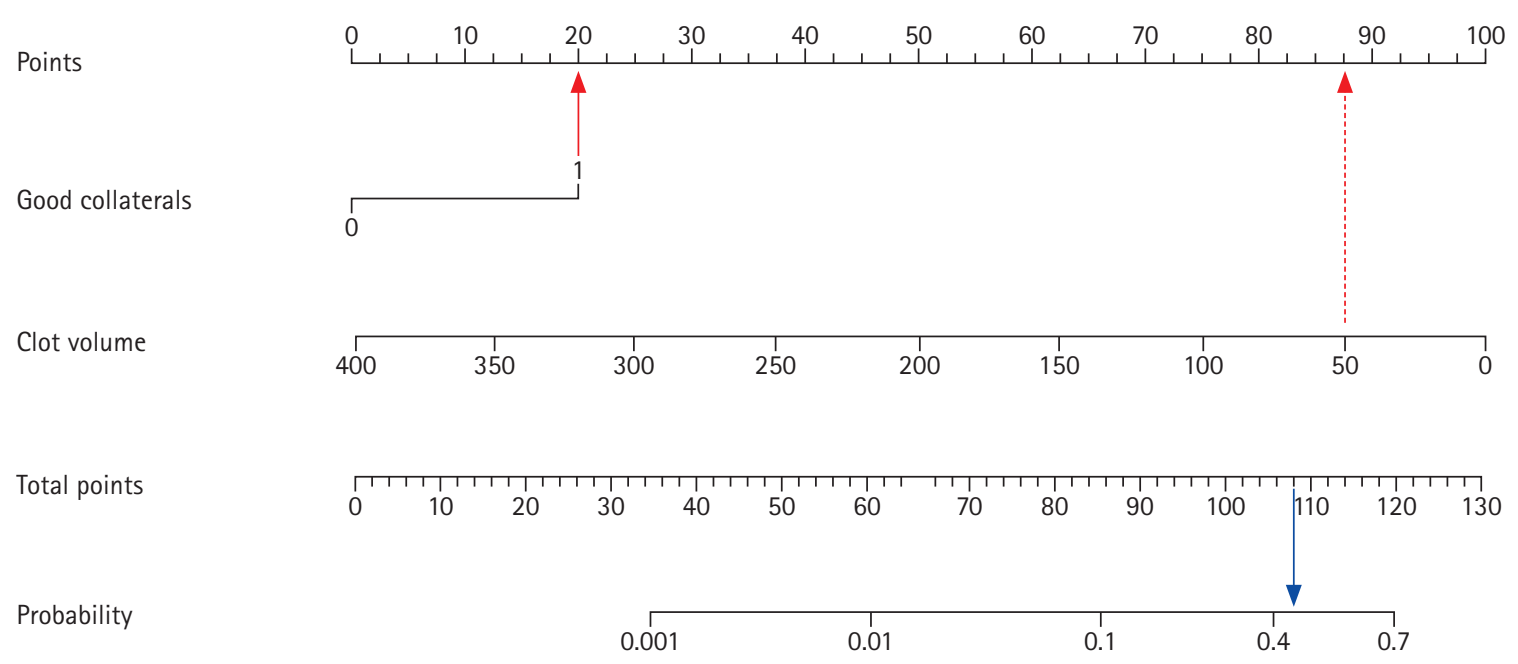

Supplementary Figure 1. Nomogram for predicting early successful recanalization after intravenous tissue plasminogen activator. When using nomogram, higher total points based on the sum of the assigned number of points for good collaterals and clot volume were associated with the chance of early recanalization. Firstly, points based on good collateral and clot volume should be calculated. Then, the summation of these points indicates the probability of the early recanalization. For example, a patient with good collaterals and clot volume of $50 \mathrm{~mm}^{3}$ would have a total of 108 points (20 points for good collaterals [red solid line] and 88 points for clot volume [red dashed line]). The predicted early successful recanalization (blue solid line) is $44.4 \%$ for this patient. 